\title{
Two Contributions of Constraint Programming to Machine Learning
}

\author{
Arnaud Lallouet and Andreï Legtchenko \\ Université d'Orléans - LIFO, \\ BP6759, F-45067 Orléans \\ lallouet, legtchen@lifo.univ-orleans.fr
}

\begin{abstract}
A constraint is a relation with an active behavior. For a given relation, we propose to learn a representation adapted to this active behavior. It yields two contributions. The first is a generic metatechnique for classifier improvement showing performances comparable to boosting. The second lies in the ability of using the learned concept in constraint-based decision or optimization problems. It opens a new way of integrating Machine Learning in Decision Support Systems.
\end{abstract}

\section{Introduction}

A constraint is a relation with an active behavior. In Constraint Programming, relations are used to model decision or optimization problems. Its success relies on two aspects: first the model is high level, declarative and easy to understand and second, there exists a range of powerful techniques to find and optimize solutions. The key concept of Constraint Programming is that relations are actively used during search for enforcing a consistency. In the context of finite domains constraints, consistencies are used to reduce variable domains in order to limit the search effort. The more the domains get reduced, the less branches are explored in the search tree. It works so well that, while theoretically intractable, many problems are practically solvable.

But the model is usually limited to relations which are completely known. In this paper, we propose to learn concepts as constraints. We call them open constraints because they are only partially known by a set of positive and negative examples. While the acquisition of such an object has been extensively studied in Machine Learning, no other work, to the authors' best knowledge, has considered to acquire the relation in such a way that consistency inference could be performed. This paper deals with the induction of a relation using a representation suitable for constraint propagation.

The first contribution is a generic meta-technique for classifier improvement. The constraint-based representation involves a decomposition of the relation in multiple parts according to some projections. Each part is learnable with classical techniques and the relation is reconstituted by a vote mechanism. By reference to Constraint Programming and because they represent a kind of internal consistency of the relation, we call the resulting representation a Consistency Checking 
Classifier. It happens that classical techniques are improved by this decomposition and the resulting combination of decisions. The improvement provided by this technique is different and orthogonal to boosting [5]. Actually, both can be combined and the best results are obtained by using both techniques at the same time.

The second contribution is the transformation of a classifier into a propagator. We show that if the relation is acquired with the suggested constraint representation, the relation can be turned into a constraint. In order to do this, the classifiers are transformed into propagators which ensure the active behavior of the constraint. This allows Machine Learning to be used in new applications when included in constraint-based decision support systems. Basically, this kind of system routinely considers millions of alternatives and tries to find a satisfactory solution. Using a relation represented by a classifier inside such a system imposes to first generate an alternative and evaluate it with the classifier. This technique, known as "generate and test", is computationally very expensive. The purpose of an active constraint is to filter wrong alternatives much earlier in the search process.

We first present a short introduction to constraint solving useful to understand the idea of consistency checking classifier and the transformation of a classifier into propagator. Section 3 is devoted to the presentation of the learning technique and its experimental evaluation. In section 4, we present shortly how to turn a classifier into propagator.

\section{Open Constraints}

First, let us set the notations we use. Let $V$ be a set of variables and $D=$ $\left(D_{X}\right)_{X \in V}$ be the family of their finite domains. For $W \subseteq V$, we denote the Cartesian product $\Pi_{X \in W} D_{X}$ by $D^{W}$. For a set $E$, we denote by $\mathcal{P}(E)$ its powerset, by $\bar{E}$ its complementary and by $|E|$ its cardinal. Projection of a tuple (or a set of tuples) on a set of variables is denoted by $\mid$. For $X \in W$, we denote by $W_{-X}$ the set $W \backslash\{X\}$.

A constraint $c=(W, T)$ is composed of a subset $W \subseteq V$ of variables and a relation $T \subseteq D^{W}$. A Constraint Satisfaction Problem (or CSP) is a set of constraints. A solution of a CSP is an assignment of the variables which satisfy all constraints. Solving a CSP means finding a solution but in some cases it is also required to find the best solution according to an external optimization criterion. Instead of searching in a space of possible assignments, a search state $s$ is composed of a set of currently possible values for each variable. Formally, $s=$ $\left(s_{X}\right)_{X \in V}$ where $s_{X} \subseteq D_{X}$. It represents the set $\Pi_{X \in V} s_{X}$. This representation is economic in term of space but the counterpart is that only Cartesian products are representable. Thus an assignment is represented by a search state in which all variable domains are singletons.

Domains are reduced by the application of propagators. A propagator for a constraint $c=(W, T)$ and a variable $X \in W$ is a function $f_{X}$ which reduces the domain $s_{X}$ of variable $X$ in such a way that no solution of $c$ included in the search 
state $s$ is lost [2]. In other words, a propagator suppresses values which do not occur in any solution: these values have no support in the constraint. The most reducing propagator is the one associated with the well-known arc-consistency. It only keeps in the domain of a variable the values which can be extended to a solution of the constraint. Propagators for all constraints are iterated up to reach their greatest fixpoint, and this defines the expected consistency. A possible way to implement a propagator is to associate a boolean function $f_{X=a}$ to every value $a \in D_{X}$. We call such a function an Elementary Reduction Function (or ERF). This function takes as input the current domain of all variables but $X$, i.e. $\left.s\right|_{W_{-X}}$, and answers true if there is a support for $X=a$ in $c$ according to the current search state $s$ and false otherwise. By applying ERFs associated to each value of the domain, we are able to reconstitute a propagator: $f_{X}\left(\left.s\right|_{W}\right)=$ $s_{X} \cap\left\{a \in D_{X} \mid f_{X=a}\left(\left.s\right|_{W_{-X}}\right)=\right.$ true $\}$. An ERF must be correct, which means that it does not suppress a value which has a support. But it can be incomplete, i.e. that an unsupported value may remain undetected if the condition verified by the ERF is too weak.

In classical Constraint Programming, all relations are completely known. If we want to use a concept known by positive and negative examples as a constraint, we have to switch to a broader setting:

\section{Definition 1 (Open Constraint).}

An open constraint is a triple $c=\left(W, c^{+}, c^{-}\right)$where $c^{+} \subseteq D^{W}, c^{-} \subseteq D^{W}$ and $c^{+} \cap c^{-}=\emptyset$.

In an open constraint $c=\left(W, c^{+}, c^{-}\right), c^{+}$represents the set of known allowed tuples (positive examples) and $c^{-}$the forbidden ones (negative examples). The remaining tuples are unknown. For an open constraint $c=\left(W, c^{+}, c^{-}\right)$, the learning task is to find a complete relation compatible with the open one, i.e. a constraint $c^{\prime}=(W, T)$ such that $c^{+} \subseteq T$ and $c^{-} \subseteq \bar{T}$. We call such a constraint $c^{\prime}$ an extension of the open constraint $c$.

\section{Consistency Checking Classifiers}

A classical learning technique for a relation consists in finding a classifier which answers true for a tuple which belongs to the relation and false otherwise. Inspired by the way constraints check their satisfiability, we propose instead to learn the projections of the relation on the hyperplane orthogonal to a value of a variable. For an open constraint $c=\left(W, c^{+}, c^{-}\right)$, a variable $X \in W$ and a value $a \in D_{X}$, this projection is defined by:

$$
c_{<X=a>}=\left(W_{-X},\left\{\left.t\right|_{W_{-X}}\left|t \in c^{+} \wedge t\right|_{X}=a\right\},\left\{\left.t\right|_{W_{-X}}\left|t \in c^{-} \wedge t\right|_{X}=a\right\}\right)
$$

Since the projection defines a new relation, we can use an arbitrary classifier to learn it. We call it an elementary classifier by analogy with ERFs. We build an elementary classifier for all values in the domain of all variables and we call this set an consistency checking classifier: 


\section{Definition 2 (Consistency Checking Classifier).}

Let $c=\left(W, c^{+}, c^{-}\right)$be an open constraint, $X \in W, a \in D_{X}$ and let $c l_{<X=a>}$ be a classifier for the relation $c_{<X=a>}$. A consistency checking classifier (or CCC) for $c$ is the set of elementary classifiers $\left\{c l_{<X=a>} \mid X \in W, a \in D_{X}\right\}$.

Following the intuition of ERFs for solving, we can use these elementary classifiers to decide if a tuple belongs to the extension of the open constraint or not. It can be done by checking if the tuple gets rejected by the classifiers. Let $t \in D^{W}$ be a candidate tuple and let $\left(c l_{<X=\left.t\right|_{X}>}\left(\left.t\right|_{W_{-X}}\right)\right)_{X \in W}$ be the family of $0 / 1$ answers of the elementary classifiers which are concerned by the tuple. We can interpret the answers according two points of view: (a) vote with veto: the tuple is accepted if and only if accepted by all classifiers, (b) majority vote: the tuple is accepted if accepted by a majority of elementary classifiers. Many other combinations could be envisaged [13] but our aim in this article is only to prove the validity of the method, not to find the best combination of votes. In the rest of this section, we only consider majority vote.

In order to learn the projection relations, we used two types of classifiers in our experiments: a multi-layer perceptron (MLP) and the C5.0 decision tree learning algorithm. For $W \subseteq V$, a neuron is a function $n: \mathbb{R}^{|W|} \rightarrow \mathbb{R}$ computing the weighted sum of its inputs followed by a threshold sigmoid unit. A dummy input set at 1 is added to tune the threshold. Let $\left(\omega_{X}\right)_{X \in W}$ be the weights associated with each input variable and $\omega_{0}$ be the adjustment weight for the dummy input. Here is the function computed by a neuron taking as input $t=$ $\left(t_{X}\right)_{X \in W}$ :

$$
\eta(t)=\frac{1}{1+e^{\omega_{0}-\sum_{X \in W} \omega_{X} \cdot t_{X}}}
$$

For a constraint $c=\left(W, c^{+}, c^{-}\right)$, the classifier we build for $X=a$ is a tree of neurons with one hidden layer as depicted in figure 1, Let $\left(\eta_{i}\right)_{i \in I}$ be the intermediary nodes and out be the output node. All neurons of the hidden layer have as input a value for each variable in $W_{-X}$ and are connected to the output node. Let us call $n_{<X=a>}$ the network which concerns $X=a$. Since

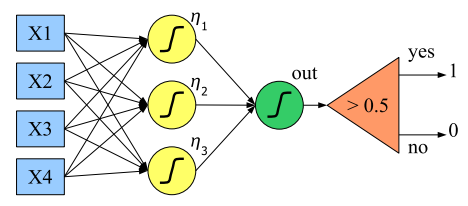

Fig. 1. Structure of the ANN neurons are continuous, we use an analog coding of the domains: $D$ is mapped on [0..1] by coding $a_{i} \in D$ by $i / n$. The output is in the interval [0..1] and we choose as convention that the value $a$ should be removed from the domain of $X$ if out $\leq 0.5$. This threshold is the last level of the network depicted in figure 1. The networks are trained by the classical backpropagation algorithm [12]. For decision trees, we use the See5.0 system [11]. This system implements the C5.0 algorithm, which is an evolution of C4.5 [9] and also the technique of boosting [5].

The classification technique has been run on a database called salad defining the concept of recipe for good salads and the following training sets from the 
UCI Machine Learning repository1: mush: mushroom, cancer: breast-cancerwisconsin, votes: house-votes-84, spam: spambase, hepat: hepatitis, cr-scr: credit screening. Continuous data are discretized such that roughly the same number of examples is put in each interval. As for boosting, the learning time is proportional to the learning time of the underlying technique (typically less than a minute for decision trees in our benchmarks). To evaluate and compare

Table 1. Learning results

\begin{tabular}{|r|r|r|r|r|r|r|r|}
\hline Database & salad & mush & cancer & votes & spam & hepat & cr-scr \\
\hline Arity & 22 & 22 & 9 & 16 & 58 & 20 & 16 \\
\hline Size of DB & 334 & 8124 & 699 & 435 & 4601 & 155 & 690 \\
\hline Type & symb & symb & symb & symb & cont & mixed & mixed \\
\hline Domain sz & $2-4$ & $2-12$ & 10 & 2 & $2-29$ & $2-4$ & $2-14$ \\
\hline \# neurons in HL & 3 & 3 & 5 & 5 & 10 & 5 & 5 \\
\hline MLP err & 3.89 & 1.75 & 4.49 & 5.78 & 10.80 & 22.07 & 16.12 \\
\hline C5.0 err & 9.86 & 1.39 & 5.46 & 3.71 & 8.65 & 20.63 & 14.35 \\
\hline C5.0b err & 4.83 & 0.31 & 3.67 & 4.37 & 5.85 & 17.23 & 12.89 \\
\hline \# classifiers & 64 & 115 & 89 & 32 & 487 & 49 & 90 \\
\hline$C C C$ (MLP) err & $\mathbf{3 . 6 4}$ & 0.81 & 3.48 & 3.77 & 7.30 & 17.30 & 15.95 \\
\hline$C C C$ (C5.0) err & 4.58 & 0.81 & $\mathbf{2 . 6 1}$ & 3.68 & 6.90 & 19.53 & 13.87 \\
\hline$C C C$ (C5.0b) err & 3.88 & $\mathbf{0 . 2 7}$ & 2.90 & $\mathbf{3 . 6 2}$ & $\mathbf{4 . 9 0}$ & $\mathbf{1 6 . 8 3}$ & $\mathbf{1 2 . 7 3}$ \\
\hline
\end{tabular}

the technique, we used a classical 10-fold cross-validation repeated 3 times. Results are shown in Table 1; the database arity (Arity); Size of DB in number of tuples (only 500 are actually considered for mushroom); Type between symbolic, continuous or mixed; the range of domain sizes of the variables (Domain sz); number of neurons in the hidden layer of the MLP (\# neurons in $H L$ ); average error ratio of the underlying techniques we used, i.e. multi-layer perceptron $(M L P)$, C5.0 and C5.0 with boosting ( $C 5.0 b)$; the number of classifiers we learn for each relation with the $\mathrm{CCC}$ technique; error rate for the consistency checking classifier on top of an underlying technique. The best result in classification is depicted in bold face. The first remark is that the CCC technique always improves its underlying technique. It also provides an improvement in classification comparable in performance to boosting, boosting being slightly better in average. But since both are usable at the same time, it appears that using CCC on top of one of the classifiers is able to outperform its underlying technique.

\section{From Classifiers to Solvers}

The second contribution consists in extending learning technique to problem solving. In order to do this, we propose to transform a consistency-checking classifier into a propagator. Then, a learned concept can be used for problem

\footnotetext{
${ }^{1}$ http://www.ics.uci.edu/ ${ }^{\sim m l e a r n}$
} 
solving. While a classifier gives an answer for a single tuple, a propagator gives an answer for a Cartesian product. Since a propagator should never reject a solution, its answer should be false only if the absence of solution in the search space is proven. If there is a solution or if the absence of solution cannot be ensured because a full computation would be too expensive, then the answer has to be true. Because of the independent schedule of propagators [2], the learned relation is the one obtained in veto mode. It yields that the concept is more centered on positive examples, which is an advantage in optimization problems.

The simplest way to know if there is a solution in such a search space is to apply the elementary classifier on each tuple and combine all results with a disjunction. Unfortunately, this solution is computationally intractable for reasonable constraint arity or domain size. Another idea could be to first generate off-line the solutions of the extension of the constraint and use them for solving with a standard but efficient arc-consistency propagation algorithm like GACschema 4]. Unfortunately again, the generation time and representation size are prohibitive. Since actually covering the whole Cartesian product is impossible, the intuition of the method is to cover it virtually. In order to do this, we have to find a cheap sufficient condition to ensure that a sub-space does not contain any solution. We expose on two examples the cases of decision trees and multi-layer perceptrons.

Example 1 (Decision tree). Consider the decision tree depicted in figure 2 and assume that it is associated with the value $X=1$. The domain of the other variables are $(Y \mapsto\{0,1,2,3\}, Z \mapsto\{0,1\}, T \mapsto\{2,3\})$. In order to know if

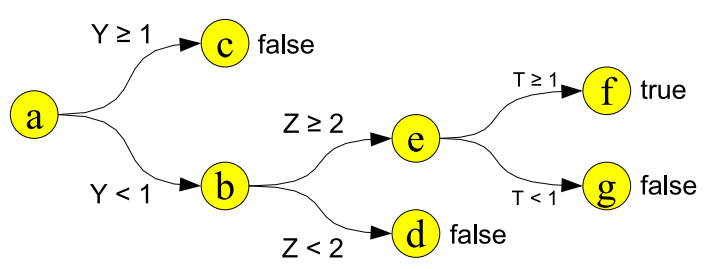

Fig. 2. A decision tree

there is a solution in this space, we define an output value $\operatorname{eval}(n)$ for a node $n$ of the tree. We start from node $a$ and evaluate the conditions to get to the children nodes $b$ and $c$. Both conditions are satisfied by some element of the search space, so the result will be the disjunction of the evaluation of both nodes: $\operatorname{eval}(a)=\operatorname{eval}(b) \vee \operatorname{eval}(c)$. In order to evaluate $b$, we test the conditions of the children nodes $d$ and $e$. Since the condition for node $e$ is not met, we can stop here the evaluation of the subtree and return eval $(e)=$ false. Since $\operatorname{eval}(d)=$ false, we can complete the evaluation of node $b$ with $\operatorname{eval}(b)=\operatorname{eval}(d) \vee \operatorname{eval}(e)=$ false $\vee$ false $=$ false. Back to $a$, we output $\operatorname{eval}(a)=$ false $\vee$ false $=$ false. The answer of the classifier is the answer of the root of the decision tree. It yields that value 1 should be suppressed from $X$ 's domain. 
This transformation of a function from set to powerset is known as extension to sets [14. The case of decision trees is particularly interesting since it allows a fast computation of this extension. This is not the case for arithmetic functions, but they can be given a less precise extension called extension to intervals [8]. We call $I n t_{\mathbb{R}}$ the interval lattice built on the set $\mathbb{R}$ of real numbers. First, all functions have extensions to intervals. Let $f: \mathbb{R} \rightarrow \mathbb{R}$ be a function. A function $F: I n t_{\mathbb{R}} \rightarrow I n t_{\mathbb{R}}$ is an extension to intervals of $f$ if $\forall I \in I n t_{\mathbb{R}}, \forall x \in I, f(x) \in$ $F(I)$. An extension $F$ is monotonic if $A \subseteq B \Rightarrow F(A) \subseteq F(B)$. Between all extensions to intervals of $f$, there is a smallest one, called canonical extension to intervals: $\widehat{f}(I)=[\{f(x) \mid x \in I\}]$. The canonical extension is monotonic. Here are the canonical extensions to intervals of the operators used in perceptrons:

$$
\begin{aligned}
{[a, b]+[c, d] } & =[a+c, b+d] \\
{[a, b] \times[c, d] } & =[\min (P), \max (P)] \text { where } P=\{a c, a d, b c, b d\} \\
\exp ([a, b]) & =[\exp (a), \exp (b)]
\end{aligned}
$$

Division is not a problem in our setting since no interval contains 0 (see the sigmoid denominator). If $e$ is an expression using these operators and $E$ the same expression obtained by replacing each operator by a monotonic extension, then $\forall I \in \operatorname{Int}_{\mathbb{R}}, \forall x \in I, e(x) \in E(I)$. This property of monotonic extensions is called "The Fundamental Theorem of Interval Arithmetic" [8]. It also holds when domains are replaced by cartesian products of intervals. By taking the canonical extension of all basic operators in an expression $e$, we do not always obtain an extension $E$ which is canonical. We instead call it the natural extension.

Example 2 (Multi-layer perceptron). The multi-layer perceptron $n_{<X=a>}$ defines naturally a boolean function of its input variables. Let $N_{<X=a\rangle}$ be its natural interval extension, defined by taking the canonical extension of each basic operator,,$+- \times, /$, exp. Then, by using as input the current domain of the variables, we can obtain a range for its output. In order to do this, we compute the interval range of every neuron of the hidden layer and we use these results to feed the output neuron and compute its domain. Since we put a 0.5 threshold after the output neuron, we can reject the value $a$ for $X$ if the maximum of the output range is less than 0.5 , which means that all tuples are rejected in the current domain intervals. Otherwise, the value remains in the domain.

The extension (to sets or to intervals) $C L_{<X=a>}$ of the classifier $c l_{<X=a>}$ is an ERF. The resulting propagator defines a consistency for the constraint. We have also experimented the resulting consistencies. Decision trees and multilayer perceptrons provide a consistency weaker than arc-consistency but faster to evaluate. While arc-consistency could theoretically be obtained for some decision trees, it is not generally the case. Related work in constraint acquisition does not provide any technique for learning a constraint and building its propagator at the same time 3, 1, 6, 10. The same holds for the work in Inductive Logic Programming [7] since Prolog evaluation implements generate and test. On the other hand the logic program representation is even more concise than ours. 


\section{Conclusion}

In this paper, we propose a generic meta-technique for learning a relation and a way to use Machine Learning techniques in decision or optimization problems. The first involves a decomposition of the relation in projections and improves the performances in classification. The second consists in the transformation of the classifier into a constraint propagator, which allows to use the classifier on sub-spaces instead of only tuples. We hope this work will foster cross-fertilization between these two fields.

\section{References}

1. Slim Abdennadher and Christophe Rigotti. Automatic generation of rule-based constraint solvers over finite domains. ACM TOCL, 5(2), 2004.

2. K.R. Apt. Principles of Constraint Programming. Cambridge University Press, 2003.

3. K.R. Apt and E. Monfroy. Automatic generation of constraint propagation algorithms for small finite domains. In Int. Conf. on Constraint Programming, volume 1713 of $L N C S$, pages 58-72. Springer, 1999.

4. Christian Bessière and Jean-Charles Régin. Arc-consistency for general constraint networks: preliminary results. In IJCAI, pages 398-404, Nagoya, Japan, 1997. Morgan Kaufmann.

5. Y. Freund and R. Shapire. A short introduction to boosting. Journal of Japanese Society for Artificial Intelligence, 14(5):771-780, 1999.

6. Arnaud Lallouet, Thi-Bich-Hanh Dao, Andreï Legtchenko, and AbdelAli Ed-Dbali. Finite domain constraint solver learning. In Georg Gottlob, editor, International Joint Conference on Artificial Intelligence, pages 1379-1380, Acapulco, Mexico, 2003. AAAI Press.

7. Nada Lavrac and Saso Dzeroski. Inductive Logic Programming: Techniques and Applications. Ellis Horwood, 1994.

8. Ramon E. Moore. Interval Analysis. Prentice Hall, 1966.

9. J. Quinlan. C4.5: Programs for Machine Learning. Morgan Kaufmann, 1993.

10. F. Rossi and A. Sperduti. Acquiring both constraint and solution preferences in interactive constraint system. Constraints, 9(4), 2004.

11. RuleQuest Research. See5: An informal tutorial, 2004. http://www.rulequest.com/ see5-win.html.

12. D.E. Rumelhart, G.E. Hinton, and R.J. Williams. Learning internal representations by error propagation. Parallel Distributed Processing, vol 1:318-362, 1986.

13. Grigorios Tsoumakas, Ioannis Katakis, and Ioannis P. Vlahavas. Effective voting of heterogeneous classifiers. In J.-F. Boulicaut, F. Esposito, F. Giannotti, and D. Pedreschi, editors, ECML, volume 3201 of $L N C S$, pages 465-476, Pisa, Italy, September 20-24 2004. Springer.

14. R. C. Young. The algebra of multi-valued quantities. Mathematische Annalen, 104:260-290, 1931. 